\title{
Gamma-Hydroxybutyrate Measurement
}

National Cancer Institute

\section{Source}

National Cancer Institute. Gamma-Hydroxybutyrate Measurement. NCI Thesaurus. Code C75357.

The determination of the amount of gamma-hydroxybutyrate (GHB) present in a sample. 